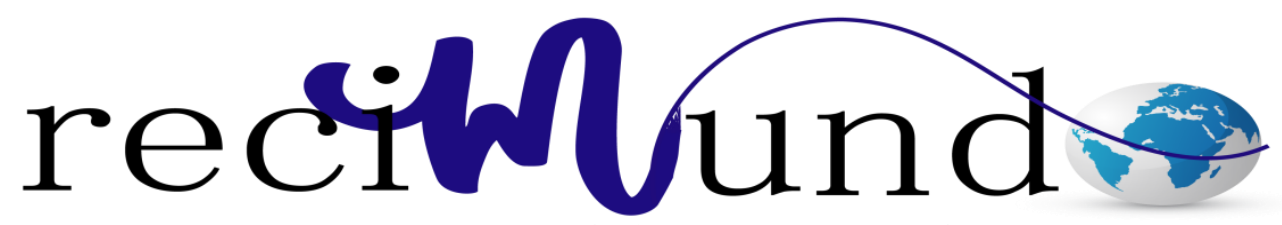

Revista Cientifica Mundo de la Investígación y el Conocimiento

Carlos Gonzalo Aguirre Rodríguez ${ }^{\text {a }}$

La inocuidad en el proceso productivo del cacao en las Mipymes

Revista Científica Mundo de la Investigación y el Conocimiento. Vol. 1 núm., 5, diciembre, 2017, pp. 52-63

DOI: $10.26820 /$ recimundo/1.5.2017.52-63

Editorial Saberes del Conocimiento 


\section{La inocuidad en el proceso productivo del cacao en las Mipymes}

Vol. 1, núm. 5., (2017)

Carlos Gonzalo Aguirre Rodríguez

\section{RESUMEN}

El presente estudio de investigación tiene por objetivo probar que la inocuidad, referida a la seguridad que presenta un producto al momento del consumo humano, en el proceso productivo del cacao en las Mipymes influye en su mejora de manera continua, centrándose en la revisión y compilación de información pertinente, considerando publicaciones importantes, por tanto se trata de una revisión bibliográfica de referencia, no experimental, a través de la cual se encontró que existe una relación estrecha entre la gestión de la calidad, estrictamente vinculada a la inocuidad en el proceso de producción del cacao y la mejora continua del mismo, tomando en cuenta principalmente los aportes de las normas para el control de la calidad ISO 22000, de la Organización Mundial de la Salud, literaturas relacionadas con la economía y el control de calidad en procesos de producción y artículos científicos disponibles. Así mismo, se valoran los procesos productivos con los siguientes indicadores: buenas prácticas de manufactura, análisis de peligro y puntos críticos de control, benchmarking, que tienen relación directa con la inocuidad dentro del proceso. Concluyendo que la intervención de la Gestión de calidad, a través de los indicadores de gestión de calidad, los cuales están íntimamente relacionados con la inocuidad de los productos, influyen en el control y mejora de los procesos productivos en las pymes de industrialización del cacao.

Palabras claves: Inocuidad; mejora; proceso; calidad; cacao. 


\section{La inocuidad en el proceso productivo del cacao en las Mipymes}

Vol. 1, núm. 5., (2017)

Carlos Gonzalo Aguirre Rodríguez

\section{ABSTRACT}

The objective of this research study is to prove that safety, referring to the safety of a product at the time of human consumption, in the production process of cocoa in MIPYMES influences its improvement continuously, focusing on the review and compilation of relevant information, considering important publications, therefore it is a bibliographic review of reference, not experimental, through which it was found that there is a close relationship between quality management, strictly linked to safety in the process of production of cocoa and continuous improvement, taking into account mainly the contributions of the standards for the control of ISO 22000 quality, literature related to economics and quality control in production processes and scientific articles available. Likewise, the productive processes are valued with the following indicators: good manufacturing practices, hazard analysis and critical control points, benchmarking, which are directly related to safety within the process. Concluding that the intervention of Quality Management, through the indicators of quality management, which are closely related to the safety of the products, influence the control and improvement of production processes in the SMEs of cocoa industrialization.

Keywords: Safety; improvement; process; quality; cocoa. 


\section{La inocuidad en el proceso productivo del cacao en las Mipymes}

Vol. 1, núm. 5., (2017)

Carlos Gonzalo Aguirre Rodríguez

\section{Introducción.}

La gestión de calidad, consiste en aplicar el concepto de "Calidad Total" a los sistemas de gestión de la empresa. Con ello lo que se pretende es integrar la calidad en todos los procesos de la organización, establece unos principios básicos para poder implementarla en una organización. Estos son: La calidad es lo primero, el cliente como una prioridad, tratar de tomar decisiones justificadas mediante hechos y datos, hacer una priorización de actividades, control en origen de la actividad y tener un trato respetuoso hacia las personas. (Hernández Sampieri, 2014)

Dentro de este marco epistemológico tenemos cinco grandes pensadores y filósofos como son: Según (Deming, 1950) aporto una serie de puntos los cuales debemos adoptarlos como una filosofía de vida para una empresa ya sea pequeña, mediana o grande o simplemente desarrollarlos en tu vida frecuente y así lograr una mejor calidad de vida.

(Juran, 1994) Señala que la administración para lograr calidad abarca tres procesos básicos: la planificación de la calidad, el control de la calidad y el mejoramiento de la calidad.

Según (Crosby, 1952) la calidad se basa en la prevención de prevenir errores basados en la conformidad. Es decir, los requerimientos tienen que estar claramente establecidos para que no hayan malentendidos; las mediciones deben ser tomadas continuamente para determinar conformidad con esos requerimientos; la no conformidad detectada es una ausencia de calidad". En resumen, podemos decir que calidad es: Cumplir con los requerimientos que necesita el cliente con un mínimo de errores y defectos. Ya que, su pensamiento filosófico se determina en lo siguiente:

- La calidad se define como cumplimiento de requisitos

- El sistema de calidad es prevención

- El estándar de realización es cero defectos

- La medida de la calidad

La tendencia en el presente siglo es que las empresas deben actualizarse en base al escenario de la globalización ya que a la calidad anteriormente no le daban la importancia del 


\section{La inocuidad en el proceso productivo del cacao en las Mipymes}

Vol. 1, núm. 5., (2017)

Carlos Gonzalo Aguirre Rodríguez

caso, pero en el presente siglo tiene un papel importante porque el cliente pide muchas comodidades y beneficios para poder seguir consumiendo un determinado producto o servicio. Y esto se consigue por medio de una buena inversión y los buenos liderazgos en las diferentes empresas.

La inocuidad de los alimentos se ha convertido en un punto de referencia mundial para los gobiernos, los productores y consumidores de alimentos, por ser un factor determinante para ser competitivos en los mercados mundiales que demandan productos de consumo humano, así mismo, los consumidores exigen productos seguros e inocuos y una adecuada trazabilidad en el manejo de los alimentos. Es decir, La inocuidad de los alimentos se refiere a la existencia de peligros asociados a los alimentos en el momento de su consumo (ingestión por los consumidores). Como la introducción de peligros para la inocuidad de los alimentos puede ocurrir en cualquier punto de la cadena alimentaria, es esencial un control adecuado a través de toda la cadena alimentaria. Así, la inocuidad de los alimentos está asegurada a través de la combinación de esfuerzos de todas las partes que participan en la cadena alimentaria. (ISO 22000, 2005)

En la actualidad, uno de los problemas complejos que afrontan las Mipymes de industrialización del cacao en Ecuador, es el persistente riesgo epidemiológico inherente al producto alimentario procesado: comprende alimentos que por su naturaleza, composición, proceso, manipulación y población a la que va dirigida tiene alto, mediano y bajo riesgo de causar daño a la salud. Ya que, los consumidores exigen cada vez, más atributos de calidad en los productos que adquieren, siendo una característica esencial e implícita la inocuidad (apto para el consumo humano). Por otro lado, el sector alimentario trata de bajar los costos de producción y venta, las Buenas Prácticas de Manufacturas (BPM) ofrecen la posibilidad de lograrlo manteniendo la calidad y asegurando la inocuidad. Además de ser obligatorias, algunas prácticas llevan a importantes mejoras y no requieren la inversión de capital, en especial cuando hablamos del orden, la higiene y la capacitación del personal.

La gestión de calidad de las Mipymes en el proceso de producción de cacao son incipientes en la mejora continua, esta situación es consecuencia de las deficiencias en el desarrollo 


\section{La inocuidad en el proceso productivo del cacao en las Mipymes}

Vol. 1, núm. 5., (2017)

Carlos Gonzalo Aguirre Rodríguez

industrial que se dado a mediano y largo plazo y de la toma de decisiones sin un previo análisis sobre gestión de calidad generando falta de competitividad global. Ya que, la falta de concientización de los directivos acerca de la importancia de las normas de calidad y de su aporte a los procesos productivos, permite producir con baja calidad teniendo como resultado estancamientos de los parámetros de productividad. Además, la falta de personal preparado que requiere conocimiento de métodos apropiados para desarrollar procesos productivos acorde a una normatividad de calidad, ha creado un deterioro del desarrollo tecnológico de las pequeñas y medianas empresa de industrialización del cacao en el Ecuador. Por lo tanto, esta situación indica que la gestión de calidad se debe impulsar para el desarrollo de procesos productivos con enfoque hacia la competitividad de las pequeñas y medianas empresa de industrialización del cacao y mejora continua.

La Norma de la familia ISO, que específica los requisitos para un sistema de Inocuidad de los alimentos está estructurada de la siguiente manera:

Apartados del 1 al 3: Objeto y campo de aplicación, referencias normativas, términos y definiciones

Apartado 4: Sistema de gestión de la inocuidad de los alimentos

4.1 Requisitos generales

4.2 Requisitos de documentación

- Generalidades

- Control de los documentos

- Control de los registros

Por otra parte, las buenas prácticas de manufacturas para los alimentos procesados son aplicables a los establecimientos donde se procesen, envasen y distribuyan alimentos. Así como también a los equipos, utensilios y personal manipulador sometidos al Reglamento de Registro y Control Sanitario. Es decir, a todas las actividades de fabricación, procesamiento, preparación, envasado, empacado, almacenamiento, transporte, distribución y comercialización de alimentos 


\section{La inocuidad en el proceso productivo del cacao en las Mipymes}

Vol. 1, núm. 5., (2017)

Carlos Gonzalo Aguirre Rodríguez

en el territorio nacional. A los productos utilizados como materias primas e insumos en la fabricación, procesamiento, preparación, envasado y empacado de alimentos de consumo humano. (DECRETO EJECUTIVO 3253, 2002)

La producción está estructurada de la siguiente manera:

Producción primaria

Objetivos:

La producción primaria deberá realizarse de manera que se asegure que el alimento sea inocuo y apto para el uso al que se destina. En caso necesario, esto comportará:

- Evitar el uso de zonas donde el medio ambiente represente una amenaza para la inocuidad de los alimentos;

- Controlar los contaminantes, las plagas y las enfermedades de animales y plantas, de manera que no representen una amenaza para la inocuidad de los alimentos;

- Adoptar prácticas y medidas que permitan asegurar la producción de alimentos en condiciones de higiene apropiadas.

Es imperioso reducir la probabilidad de que se origine un peligro que pueda menoscabar la inocuidad de los alimentos o su aptitud para el consumo en etapas posteriores de la cadena alimentaria.

- Higiene del medio

- Producción higiénica de materias primas

- Manipulación, almacenamiento y transporte

- Saneamiento, mantenimiento de las instalaciones e higiene del personal en la producción primaria.

- La función del gobierno

(Sánchez, 1999) en su Tesis la Seguridad Alimentaria en el Ecuador, recomienda al gobierno de nacional para el desarrollo de políticas de seguridad alimentaria a nivel nacional en 


\section{La inocuidad en el proceso productivo del cacao en las Mipymes}

Vol. 1, núm. 5., (2017)

Carlos Gonzalo Aguirre Rodríguez

cuanto a la calidad e inocuidad de los alimentos, la reestructuración del sistema de control de la calidad en la producción de alimentos que favorezca la exportación y beneficie el consumo interno y la Obligatoriedad del etiquetado nutricional.

\section{Materiales y Métodos}

Bajo este escenario, la academia logra disponer de publicaciones valiosas a través de los filtros que el mismo investigador pueda desarrollar, para ello las ciencias metodológicas registra una necesidad de producir compendios de valor científico por medio de las revisiones bibliográficas documentales.

La presente investigación se ha centrado en la revisión y compilación de información pertinente para determinar la influencia de la inocuidad en el proceso productivo del cacao en las Mipymes en su mejora continua, considerando publicaciones importantes e interesantes, por tanto se trata de una revisión bibliográfica de referencia, no experimental.

La revisión bibliográfica y documental constituye uno de los principales pilares en los que se sustenta la investigación educativa. La elaboración del marco teórico a partir de la revisión documental resulta imprescindible, ya que, fundamentalmente, nos permite delimitar con mayor precisión nuestro objeto de estudio y constatar el estado de la cuestión, evitando así volver a descubrir la rueda, es decir, evitar resolver un problema que ya ha sido resuelto con anterioridad por otros investigadores.

Del mismo modo, esta revisión de la literatura permite al investigador establecer la importancia del estudio que pretende desarrollar y, posteriormente, comparar sus resultados con los de otros estudios similares. (Rodríguez \& Jordi Valldeoriola Roquet, 2012)

En éste orden de ideas, la presente investigación concluye en un compendio teórico preventivo útil y sencillo para precisamente atender el desinterés respecto a como influye la inocuidad en el proceso productivo del cacao en las Mipymes en su mejora continua, tomando en cuenta principalmente los aportes de las normas para el control de la calidad ISO 22000, de la 


\section{La inocuidad en el proceso productivo del cacao en las Mipymes}

Vol. 1, núm. 5., (2017)

Carlos Gonzalo Aguirre Rodríguez

Organización Mundial de la Salud, literaturas relacionadas con la economía y el control de calidad en procesos de producción y artículos científicos disponibles.

\section{Resultados}

En la actualidad, uno de los problemas complejos que afrontan las Mipymes de industrialización del cacao en Ecuador, es el persistente riesgo epidemiológico inherente al producto alimentario procesado: comprende alimentos que por su naturaleza, composición, proceso, manipulación y población a la que va dirigida tiene alto, mediano y bajo riesgo de causar daño a la salud. Ya que, los consumidores exigen cada vez, más atributos de calidad en los productos que adquieren, siendo una característica esencial e implícita la inocuidad (apto para el consumo humano). Por otro lado, el sector alimentario trata de bajar los costos de producción y venta, las Buenas Prácticas de Manufacturas (BPM) ofrecen la posibilidad de lograrlo manteniendo la calidad y asegurando la inocuidad. Además de ser obligatorias, algunas prácticas llevan a importantes mejoras y no requieren la inversión de capital, en especial cuando hablamos del orden, la higiene y la capacitación del personal.

La gestión de calidad de las Mipymes en el proceso de producción de cacao son incipientes en la mejora continua, esta situación es consecuencia de las deficiencias en el desarrollo industrial que se dado a mediano y largo plazo y de la toma de decisiones sin un previo análisis sobre gestión de calidad generando falta de competitividad global. Ya que, la falta de concientización de los directivos acerca de la importancia de las normas de calidad y de su aporte a los procesos productivos, permite producir con baja calidad teniendo como resultado estancamientos de los parámetros de productividad. Además, la falta de personal preparado que requiere conocimiento de métodos apropiados para desarrollar procesos productivos acorde a una normatividad de calidad, ha creado un deterioro del desarrollo tecnológico de las pequeñas y medianas empresa de industrialización del cacao en el Ecuador. Por lo tanto, esta situación indica que la gestión de calidad se debe impulsar para el desarrollo de procesos productivos con enfoque hacia la competitividad de las pequeñas y medianas empresa de industrialización del cacao. 


\section{La inocuidad en el proceso productivo del cacao en las Mipymes}

Vol. 1, núm. 5., (2017)

Carlos Gonzalo Aguirre Rodríguez

Además, el estado ecuatoriano presenta pocas políticas de incentivos y protección adecuadas para fomentar a las pequeñas y medianas empresas de industrialización del cacao tomando en consideración que la marca de Ecuador es el cacao. Actualmente las Mipymes carecen de un sistema de tecnología apropiado que ayude a mejorar su desarrollo industrial y poder realizar una integración vertical entre la producción primaria y el procesamiento. Por lo tanto, el estado debe estimular la productividad y competitividad de las pequeñas y medianas de industrialización del cacao, mediante el crecimiento de aprendizaje de las normas de calidad en el talento humano, para lograr en la medida un mayor desarrollo tecnológico y se vuelvan competitivas las Mipymes.

La inocuidad es un atributo esencial a asegurar en un alimento, lo que permite definirlo como "inofensivo, seguro, que no produce daño a la salud"; es, por tanto, el aspecto cualitativo más importante a brindar y muy exigido por los consumidores de hoy (calidad higiénica o sanitaria). (Siqueira, 2003)

La práctica de la logística tiene un impacto directo sobre la inocuidad de los productos: un inadecuado control de recepción, el ingreso de materiales sin controles de calidad sin registros ni certificados de análisis, de dudosa calidad, la recepción de un material indeseado, peligro de uso equivocado, la imposibilidad de garantizar trazabilidad posterior, un transporte inadecuado, contaminación física directa o cruzada, degradación del material - Crecimiento microbiano, almacenamiento inadecuado: Vencimientos-Contaminación por mezcla, alteraciones del material - Accidentes de trabajo- 'Daño al medio ambiente, hasta una incorrecta gestión de sock: StockOut: compras de emergencia- uso de material alternativo no adecuado. (Siqueira, 2003)

\section{Conclusiones}

A continuación se puntualizan aspectos concluyentes de la investigación:

- El sistema sanitario de las Mipymes adolecen de un insuficiente nivel de cobertura. Es decir, un sistema estático y desactualizado, en el cual no existe una revisión permanente de la normatividad sanitaria, no dispone de información, capacitación para implementar un programa de calidad, lo cual no garantiza el cumplimiento de las más mínimas 


\section{La inocuidad en el proceso productivo del cacao en las Mipymes}

Vol. 1, núm. 5., (2017)

Carlos Gonzalo Aguirre Rodríguez

condiciones sanitarias. Por lo tanto, las áreas en que más apoyo requiere son las de tecnologías y sistema de calidad e inocuidad de los alimentos.

- En la implementación de sistemas como el HACCP y de BPM se han registrado ciertos progresos a nivel de las Mipymes, los cuales son aún incipientes. A pesar de estos avances aún queda mucho trabajo por hacer en esta área, la inocuidad cobra especial importancia en el momento del pos cosecha. Sin embargo, se le ha dado poco interés al manejo de la pos cosecha y al correcto almacenamiento de los productos.

\section{Recomendaciones}

- En virtud de las insuficiencias por cubrir temas relacionados con la integración de las Buenas Prácticas de Manufactura, análisis de peligros, puntos críticos de control y prevención de riesgos; manejo de la inocuidad de los productos durante el proceso productivo, específicamente en la obtención del cacao por parte de las Mypmes, se recomienda ampliar los estudios de investigación en dicha área con la finalidad de cubrir los vacíos existentes y mejorar de manera continua la calidad de los productos Ecuatorianos (cacao) por parte de los micro, pequeños y medianos empresarios.

- Se aconseja mantener un sistema integral de comunicación de enlace, caracterizado por ser claro, preciso y a tiempo, con quienes hacen uso de la metrología en las operaciones de los procesos, además brindar capacitación sobre cómo utilizar los estándares de producción y cuáles son los riesgos de una manipulación errónea que pone en riesgo la inocuidad del producto, aspectos que pueden ser dados en forma personal y por medio de la tecnología (internet).

- Finalmente se debe tomar conciencia que implantar un Sistema de planificación de Calidad y un programa de las cinco "S" bajo la norma de inocuidad no marca el fin del objetivo sino es el principio de un proceso de mejora continua que involucra el compromiso de todos y cada uno de los miembros de la Mipymes. 


\section{Bibliografía.}

Crosby, P. B. (01 de Septiembre de 1952). www. gestiopolis.com. Recuperado el 26 de febrero de 2016, de http://www.gestiopolis.com/calidad-concepto-y-filosofias-deming-juranishikawa-y-crosby/

DECRETO EJECUTIVO 3253. (04 de Noviembre de 2002). www.epmrq.gob.ec. Recuperado el 20 de febrero de 2016, de www.epmrq.gob.ec/images/total/leyes

Deming, W. (10 de Octubre de 1950). www.gestiopolis.com. Obtenido de http://www.gestiopolis.com/calidad-concepto-y-filosofias-deming-juran-ishikawa-ycrosby/

Hernández Sampieri, R. (2014). METODOLOGIA DE LA INVESTIGACION (Sexta Edicion ed.). Mexico: McGRAW HILL.

ISO 22000. (01 de setiembre de 2005). www.iso.org. Recuperado el 20 de agosto de 2016, de www.iso.org

Juran, J. M. (06 de Febrero de 1994). www.pablogiugni.com. Recuperado el Diciembre14 de 2016, de http://www.pablogiugni.com.ar/httpwwwpablogiugnicomarp95/

Rodríguez, D., \& Jordi Valldeoriola Roquet. (2012). http://www. aliatuniversidades. com. $m x /$ bibliotecasdigitales/pdf/axiologicas/Metodologia_de_la_investigacion. pdf. Recuperado el 24 de julio de 2017, de http://www. aliatuniversidades. com. $\mathrm{mx} /$ bibliotecasdigitales/pdf/axiologicas/Metodologia_de_la_investigacion. pdf.

Sánchez, M. (1999). La Seguridad Alimentaria en el Ecuador. Quito, Ecuador: Quito/IAEN/1999.

Siqueira, R. (01 de enero de 2003). http://www.logisticamx.enfasis.com/notas/3749-procesoslogisticos-e-inocuidad-alimentaria. Recuperado el 10 de julio de 2017, de http://www.logisticamx.enfasis.com/notas/3749-procesos-logisticos-e-inocuidadalimentaria 